\title{
Interaction between BCL2 and Interleukin-10 Gene Polymorphisms Alter Outcomes of Diffuse Large B-Cell Lymphoma following Rituximab Plus CHOP Chemotherapy
}

\author{
Yeon Hee Park, ${ }^{1,2}$ Sang Kyun Sohn, ${ }^{3}$ Jong Gwang Kim, ${ }^{3}$ Myung-Hoon Lee, ${ }^{4}$ Hong Suk Song, ${ }^{5}$ \\ Min Kyoung Kim, ${ }^{6}$ Joo Seop Jung, ${ }^{7}$ Je-Joong Lee, ${ }^{8}$ Hyeoung Joon Kim, ${ }^{8}$ and Dong Hwan Kim ${ }^{1,3}$
}

\begin{abstract}
Purpose: Rituximab may overcome bcl-2-mediated chemoresistance through the inhibition of interleukin-10 (IL-10)-mediated loops, thus down-regulating bcl-2 expression. We examined the effects of genetic variation in BCL2/IL10 gene loops on treatment outcomes of diffuse large B-cell lymphoma when treated with either $\mathrm{CHOP}$ or rituximab plus $\mathrm{CHOP}$ (R-CHOP) chemotherapy. Experimental Design: Four genotypes were tested including BCL2 -938 C>A (rs2279115), $+21 \mathrm{~A}>\mathrm{G}$ ( $\mathrm{rs1801018)}$, IL10 -819 T>C (rs1800871), and -592 A>C (rs1800872) in patients receiving either R-CHOP $(n=125)$ or CHOP $(n=110)$.

Results: IL10 SNPs, -819 TT/TC or -592 AA/AC genotypes correlated with improved CHOP response rates $(P=0.04)$. Neither polymorphism separately influenced the failure-free survival (FFS) or overall survival in patients, but the $I L 10$ haplotype was associated with treatment outcomes after R-CHOP for FFS $(P=0.03)$ or progression $(P=0.007)$, whereas the -938 AA $B C L 2$ genotype significantly affected overall survival $(P=0.04)$. An interactive effect between $B C L 2$ and IL10 SNPs was significant in the group with both -938 AA BCL2 genotype and 1 to 2 copies of CC IL10 haplotype. This group showed a better FFS $(P=0.01)$ and a lower probability of progression $(P=0.004)$ compared with other genotype groups when treated with $\mathrm{R}-\mathrm{CHOP}$ chemotherapy.

Conclusions: These data indicated that R-CHOP chemotherapy resistance in diffuse large B-cell lymphoma may involve interactions between the BCL2 and IL10 genes.
\end{abstract}

The addition of rituximab to CHOP chemotherapy (R-CHOP) has significantly improved the chemotherapy response rates and survival times in patients with diffuse large B-cell lymphoma (DLBCL) compared with CHOP therapy alone $(1-5)$. However, the mechanism of action of rituximab against DLBCL has not been fully elucidated (6). The bcl-2-mediated mechanism of chemoresistance has been proposed as target of R-CHOP $(7,8)$. bcl-2 is a member of the bcl-2 family of proteins that regulate apoptosis or programmed cell death. Recent studies suggest that rituximab modulates cellular

Authors' Affiliations: 'Department of Hematology/Oncology, Samsung Medical Center, Sungkyunkwan University School of Medicine; ${ }^{2}$ Department of Hematology/Oncology, Korean Cancer Center Hospital, Seoul, Korea; Departments of ${ }^{3} \mathrm{Hematology} / O n c o l o g y$ and ${ }^{4}$ Biochemistry, Kyungpook National University Hospital; ${ }^{5}$ Department of Hematology/Oncology, Keimyung University, Dongsan Medical Center; ${ }^{6}$ Department of Hematology/Oncology, Yeungnam University, Daegu, Korea; ${ }^{7}$ Department of Hematology/Oncology, Pusal National University Hospital, Busan, Korea; and ${ }^{8}$ Department of Hematology/Oncology, Chonnam National University Hwasun Hospital, Jeollanamdo, Korea

Received 6/20/08; revised 9/11/08; accepted 9/23/08; published OnlineFirst 3/10/09.

Note: Supplementary data for this article are available at Clinical Cancer Research Online (http://clincancerres.aacrjournals.org/).

Requests for reprints: Dong Hwan Kim, Department of Hematology/Oncology, Samsung Medical Center, Sungkyunkwan University School of Medicine, Ilwon-dong 50, Kangnam-gu, Seoul, Korea 135-710. Phone: 82-53-420-6516; Fax: 82-53-426-2046; E-mail: drkiim@medimail.co.kr.

(C) 2009 American Association for Cancer Research.

doi:10.1158/1078-0432.CCR-08-1588 and molecular signal transduction pathways that regulate bcl-2-expression, particularly in patients with bcl-2-positive DLBCL (6).

Interleukin-10 (IL-10) has also been proposed as a potential prognostic marker in DLBCL patients. Previous studies have suggested that high IL-10 levels correlated with advanced stage, higher international prognostic index (IPI), and worse prognosis in DLBCL patients $(9,10)$. Serum levels of IL-10 mainly reflect tumor burden (advanced disease stage, elevated lactate dehydrogenase, and $\beta_{2}$-microglobulin; ref. 11) or enhanced activation of the immune system in patients with more aggressive disease (9).

IL-10 regulates bcl-2 expression in hematopoietic cells (12) as well as in lymphoma cells (13). Alas et al. (14-16) have suggested that inhibition of IL-10-mediated loops by rituximab could down-regulate bcl-2 expression and sensitize lymphoma cells to cytotoxic chemotherapy. Therefore, interaction between bcl-2 expression and IL-10 growth factors may contribute to the resistance mechanisms of DLBCL to chemotherapy.

Recently, various single nucleotide polymorphisms (SNP) have been identified on the genes of BCL2 and IL10. The BCL2 promoter gene SNP, the -938 C>A genotype, correlates with bcl2 expression and is associated with disease progression and survival in chronic lymphocytic leukemia (17). Lech-Maranda et al. (9) reported that the DLBCL patients with the IL10-1082G allele showed improving complete response (CR) rates as well as increased progression-free survival and overall survival (OS) times when treated with $\mathrm{CHOP}$ chemotherapy. In addition, the 


\section{Translational Relevance}

The current study investigated the predictive role of $B C L 2$ and $I L 10$ gene polymorphism on the treatment outcomes of DLBCL patients and compared their outcomes according to the chemotherapy regimen (R-CHOP versus CHOP) and BCL2/IL10 SNPs. Previous study revealed that in situ expression of BCL2 is a significant risk factor for poor outcomes following $\mathrm{CHOP}$ therapy and that R-CHOP therapy could overcome bcl-2-mediated chemoresistance. In the current study, the interaction between the BCL2 and IL10 polymorphisms on improving outcomes following $\mathrm{R}-\mathrm{CHOP}$ therapy suggests that rituximab could overcome drug resistance of DLBCL by blocking the IL-10/bcl-2-mediated loops, which could be a potential target of treatment for DLBCL. In addition, as Cerhan et al. showed that the multiple candidate gene polymorphism approach could stratify patients with follicular lymphoma according to their prognoses, the mechanism of chemoresistance in DLBCL could be investigated using the multiple candidate gene polymorphism in future, based on the current results.

IL10 gene SNPs for loci $-1082 \mathrm{~A}>\mathrm{G},-819 \mathrm{~T}>\mathrm{C}$, and $-592 \mathrm{~A}>\mathrm{C}$ showed strong correlations with progression-free or OS times in DLBCL patients receiving CHOP-based chemotherapy (9). To our knowledge, the role of BCL2 and IL10 gene polymorphisms has never been investigated in the R-CHOP therapy setting.

We hypothesized that the blocking effects of R-CHOP chemotherapy would be maximized in a milieu of high IL-10 and bcl-2 expression. We examined the clinical benefits of R-CHOP therapy in DLBCL patients with higher IL-10/bcl-2inducible genetic variants compared with a group with other IL10/bcl-2-inducible genetic variants. We also analyzed clinical outcomes in the R-CHOP $(n=125)$ and CHOP groups $(n=110)$ according to the presence of BCL2 and IL10 gene polymorphisms.

\section{Materials and Methods}

Patient characteristics and treatment protocol. A total of 235 patients who received R-CHOP $(n=125)$ or CHOP $(n=110)$ as a frontline therapy for DLBCL were included in the current study. Patient samples and clinical records were collected from six hospitals in the Republic of Korea (Kyungpook National University Hospital, Chonnam National University Hwasun Hospital, Korean Cancer Center Hospital, Pusan National University Hospital, Dongsan Medical Center, and Yeungnam Medical Center). The study was approved by the institutional research board of the Kyungpook National University Hospital.

CHOP chemotherapy was administered as follows: one course of chemotherapy consisted of intravenous infusions of cyclophosphamide $750 \mathrm{mg} / \mathrm{m}^{2}$, Adriamycin $50 \mathrm{mg} / \mathrm{m}^{2}$, vincristine $2 \mathrm{mg}$, and oral administration of $100 \mathrm{mg}$ prednisone on days 1 to 5 (CHOP). This was repeated every 3 weeks (18). The R-CHOP protocol was identical to the CHOP protocol, except for the addition of a rituximab $\left(375 \mathrm{mg} / \mathrm{m}^{2} / \mathrm{d}\right)$ intravenous infusion over 4 to $6 \mathrm{~h}$ at day 1 before administration of CHOP drugs. Patients with limited stage disease typically received 4 courses of chemotherapy followed by involved field radiotherapy
(30-40 Gy), whereas patients with advanced-stage disease received 6 to 8 courses of chemotherapy followed by radiotherapy to bulky sites. There was no maintenance treatment with rituximab after completion of the R-CHOP protocol. Twelve patients received high doses of chemotherapy with autologous stem cell rescue for relapsed disease $-(n=2)$ or as an upfront treatment for initially high IPI scores $(n=10)$. The response to $\mathrm{CHOP}$ with or without rituximab therapy was evaluated after completion of 2 to 3 courses of chemotherapy, 1 month after completion of all planned cycles of chemotherapy, and every 4 months thereafter.

Genotyping of the IL10 and BCL2 gene polymorphisms. Genomic DNA was isolated using a DNA purification kit (Bioneer) with venous blood samples in tubes with $50 \mathrm{mmol} / \mathrm{L}$ EDTA from each patient. Informed consent was obtained in all subjects. The genotypes of the IL10 promoter gene at loci $-819 \mathrm{~T}>\mathrm{C}$ ( $\mathrm{rs} 1800871)$ and $-592 \mathrm{~A}>\mathrm{C}$ (rs1800872) were assessed using the PCR with restriction fragment length polymorphisms with previously described primers (19).

The genotypes for BCL2 -938 C>A (rs2279115) and +21 A>G (rs1801018) were analyzed with rapid capillary PCR and melting curve analysis using fluorescence-labeled hybridization probes in a LightCycler System (LC480; Roche Diagnostics). The amount of fluorescence was measured using fluorescence resonance energy transfer. The PCR primer sequences and the fluorescently labeled hybridization probes are described as follows (Bioneer): for $-938 \mathrm{C}>\mathrm{A}$ genotyping (rs2279115): 5'-GCTTCACGCCTC $(\mathrm{C} \rightarrow \mathrm{A}) \mathrm{CCAG}-3^{\prime}$ (forward) $(\mathrm{C} \rightarrow \mathrm{A}$ : mismatch with NT_025028); 5'-TCCAGCAGCTTTTCGGAA-3' (reverse); 5'-CTCCGTCCCCGGCTCCTTCA-fluorescence-3' (anchor); 5'-LC640CGTCCCCTCTCCCCTGTCT-phosphate (sensor); for +21 A>G genotyping (rs1801018): 5'-GCATCCCACTCGTAGCC-3' (forward); 5'-CACCAGAATCAAGTGTTCCG-3' (reverse); 5'-TTATCGTACCCCGTTCTCCC-fluorescence-3' (anchor); 5'-LC640-GCGTGCGCCATCCTTCCC-phosphate (Sensor).

The amplification reaction was done in a final volume of $5.0 \mu \mathrm{L}$ with $1.0 \mu \mathrm{L}$ genomic DNA (10 ng), $0.5 \mu \mathrm{L}$ forward primer $(10 \mathrm{pmol} / \mu \mathrm{L})$, $0.1 \mu \mathrm{L}$ reverse primer $(10 \mathrm{pmol} / \mu \mathrm{L}), 0.25 \mu \mathrm{L}$ probe $\mathrm{FL}(2 \mathrm{pmol} / \mu \mathrm{L})$, $0.5 \mu \mathrm{L}$ probe LC640 $(2 \mathrm{pmol} / \mu \mathrm{L}), 1.0 \mu \mathrm{L}$ Roche LightCycler HybProbe reagent, $1.0 \mu \mathrm{L}$ betaine, and $0.65 \mu \mathrm{L}$ water for $-938 \mathrm{C}>\mathrm{A}$ locus. The nested reaction was in a final volume of $5 \mu \mathrm{L}$ with $1.0 \mu \mathrm{L}$ genomic DNA (10 ng), $0.2 \mu \mathrm{L}$ forward primer $(10 \mathrm{pmol} / \mu \mathrm{L}), 0.5 \mu \mathrm{L}$ reverse primer $(10 \mathrm{pmol} / \mu \mathrm{L}), 0.25 \mu \mathrm{L}$ probe FL $(2 \mathrm{pmol} / \mu \mathrm{L}), 0.5 \mu \mathrm{L}$ probe LC640 (2 pmol/ $/ \mu \mathrm{L}), 1.0 \mu \mathrm{L}$ Roche LightCycler HybProbe reagent, $1.0 \mu \mathrm{L}$ betaine, and $1.55 \mu \mathrm{L}$ water for $+21 \mathrm{~A}>\mathrm{G}$ locus. The cycling conditions were as follows: $95^{\circ} \mathrm{C}$ for $10 \mathrm{~min}$ followed by $95^{\circ} \mathrm{C}$ for $10 \mathrm{~s}, 56^{\circ} \mathrm{C}$ for $10 \mathrm{~s}$, and $72^{\circ} \mathrm{C}$ for $10 \mathrm{~s}$ repeated 45 times. The melting curve was recorded after $1 \mathrm{~min}$ denaturation $\left(95^{\circ} \mathrm{C}\right)$ followed by reannealing at $40^{\circ} \mathrm{C}$ for $1 \mathrm{~min}$ and detection during heating to $80^{\circ} \mathrm{C}$ at $0.2^{\circ} \mathrm{C} / \mathrm{s}$. The example of LightCycler-assisted analyses of the BCL2 genotyping is shown in Supplementary Fig. S1.

To confirm the genotyping results, direct sequencing was done with selected PCR-amplified DNA samples. BCL2 and IL10 genotyping has been successfully done in 216 of 235 (92\%) samples. BCL2 and IL10 genotype sequence was available in 197 samples.

Definitions. Clinical responses to CHOP with or without rituximab chemotherapy were determined by physical examination and computed tomography. Responses were scored according to the International Working Group criteria (20). OS time was measured from day 1 of the initial cycle of frontline chemotherapy to the date of death or last follow-up. Failure-free survival (FFS) time was calculated from day 1 of the initial cycle of frontline chemotherapy to treatment failure (disease progression, recurrence, or death by any cause).

Biostatistical analysis. The frequencies of BCL2 and IL10 genotypes were calculated using the haploview program (21). Haplotype frequencies were estimated with the linkage disequilibrium (LD) coefficient, $D$, using the haploview program. $D$ was expressed as $D^{\prime} . D^{\prime}$ is the value of $D$ as a percentage of the maximum calculated value based on the observed allele frequencies. Values of $D^{\prime}$ ranged between -1 and +1 . $D^{\prime}$ values of 1 denoted complete $L D$, and $D^{\prime}$ values of 0 denoted 
complete linkage equilibrium. Individual haplotype was estimated based on a Bayesian algorithm using the Phase program (22).

We hypothesized that the CC haplotype has a high IL-10 inducibility. Thus, individuals with 0 to 1 copy of the CC haplotype would have a low IL-10-inducible haplotype, whereas those with 2 copies of the CC haplotype would have a high IL-10 inducibility. In addition, the -938 AA genotype predicted high bcl-2 inducibility, whereas -938 AC/CC genotype predicted low bcl-2 inducibility. This is based on previous functional study results for the IL10 $(22-26)$ and BCL2 gene polymorphisms (17). We divided the patients into two groups based on SNP information to examine the effects of interaction between BCL2 and IL10 gene SNPs: $(a)$ the high BCL2 and high IL10 inducible group with both -938 AA BCL2 genotypes and 2 copies of CC IL10 haplotype for loci $-819 \mathrm{~T}>\mathrm{C}$ and $-592 \mathrm{~A}>\mathrm{C}\left(B C L 2^{\mathrm{High}} / I L 10^{\mathrm{High}}\right)$ and $(b)$ the low $B C L 2$ or low IL10 inducible group with -938 AC/CC BCL2 genotype or 0 to 1 copy of CC IL10 haplotype for loci -819 T>C and $-592 \quad \mathrm{~A}>\mathrm{C}$ (BCL2 ${ }^{\text {Low }}$ and/or IL10 ${ }^{\text {Low }}$ ).

Statistical analysis. Clinical data were analyzed according to information available as of December 2005. The medical records of the patients and data were reviewed retrospectively. Clinical characteristics and treatment outcomes were stratified according to the genotypes or haplotype of IL-10 promoter gene and were compared in each treatment group (R-CHOP and CHOP) using $x^{2}$, Fisher's exact, or Mann-Whitney's $U$ test. The statistical endpoints included CR, overall response rate (ORR), probability of progression, FFS, and OS.

Additive, dominant, or recessive models were analyzed by singlemarker analyses to examine the association of each genotype of IL10 or BCL2 SNPs with clinical outcomes, including CR, ORR, probability of progression, FFS, and OS. In a haplotype analysis, additive, dominant, or recessive models were also applied. Comparison of CR and ORR was done using the $x^{2}$ test, whereas the comparison of probability of progression, FFS, and OS was done using the log-rank or Wilcoxon test.

Cohorts had been previously separated into the CHOP and R-CHOP groups, and data were separately analyzed. This analysis revealed that the poor prognostic effect of bcl-2 expression was neutralized by adding rituximab to CHOP therapy $(P=0.7$ for $\mathrm{OS}$ and 0.8 for event-free survival), whereas the expression of bcl-2 was associated with poor OS when treated with CHOP therapy (8). Without separating the groups according to their frontline treatment regimens or genotypes, a sensitive signature could be neglected in evaluating the prognostic value of the candidate biomarker. Thus, we defined "stratified treatment group" as groups receiving either CHOP or R-CHOP therapy and "stratified genotype group" as groups with either BCL2 $2^{\mathrm{High}} / \mathrm{IL} 10^{\mathrm{High}}$ or BCL2 ${ }^{\mathrm{Low}}$ and/or $I L 10^{\text {Low }}$. We compared the CR, ORR, probability of progression, FFS, and OS according to (a) different genotype groups in each stratified treatment group (Supplementary Fig. S2A) and (b) between the R-CHOP and CHOP groups in each stratified genotype group (Supplementary Fig. S2B).

Further analysis was conducted to identify the predictive factors for the achievement of $\mathrm{CR}$ or ORR by multiple logistic regression analyses using the backward conditional procedure. Multivariate survival analyses using the Cox's proportional hazards model were used to define the prognostic factors for OS, FFS, or probability of progression. Backward conditional procedures were conducted until the $P$ value for the likelihood ratio test was $<0.05$ with the following variables: frontline regimen (R-CHOP versus CHOP), IPI (score 0-2 versus $3-5)$, and $B C L 2 / I L 10$ genotype $\left(B C L 2^{\mathrm{High}} / \mathrm{IL} 10^{\mathrm{High}}\right.$ versus $B C L 2^{\text {Low }}$ and/or $\left.I L 10^{\text {Low }}\right)$. The procedure was repeated in the overall population regardless of their frontline regimen and in each stratified treatment group (CHOP or R-CHOP group). The hazard ratios (HR) and 95\% confidence intervals (95\% CI) were also estimated. Statistical significance was established at $P<0.05$. The statistical data were generated using the SPSS software package (SPSS 13.0). Multivariate analyses were done on 200 of 212 patients, with 12 patients excluded due to lack of lactate dehydrogenase data at presentation.

\section{Results}

Treatment outcomes in overall group. Demographic characteristics and treatment outcomes are displayed in Table 1 . The median age was 60 years and median follow-up time was 22 months (range, 0.5-176 months) No differences in demographic characteristics were identified between the R-CHOP and the CHOP groups.

The CR rate or ORR was not significantly different between the two groups ( $P=0.2$ for $\mathrm{CR}$ and $P=0.4$ for $\mathrm{ORR}$ ) according to frontline chemotherapy. However, a significant difference was noted between the two groups with respect to FFS and the probability of progression (Supplementary Fig. S3). The 1-year FFS rate was $82.4 \pm 3.6 \%$ in the R-CHOP group and $65.7 \pm$ $4.8 \%$ in the CHOP group and 2-year FFS was $59.3 \pm 5.4 \%$ in the R-CHOP group and $54.5 \pm 5.3 \%$ in the CHOP group $(P=0.03)$. The 1-year probability of progression was $13.7 \pm$ $3.3 \%$ in the R-CHOP group and $31.3 \pm 4.8 \%$ in the CHOP group, and 2-year probability of progression was $35.8 \pm 5.5 \%$ in R-CHOP group and $41.9 \pm 5.3 \%$ in the CHOP group $(P=0.01)$. OS was not statistically different between the two groups but trended toward a survival benefit in the R-CHOP group. The 1-year OS rate was $89.4 \pm 2.9 \%$ in the R-CHOP group and $83.9 \pm 3.7 \%$ in the CHOP group and the 2 -year OS rate was $74 \pm 4.8 \%$ in the R-CHOP group and $70.9 \pm 4.9 \%$ in the CHOP group $(P=0.2)$.

Distribution of genotype and haplotype. The genotype distributions for IL10 and BCL2 gene are displayed in Table 2. The frequency of the A allele (fA) and $\mathrm{fC}$ was 0.711 and 0.289 for IL10, -592 A $>C$ genotype (Hardy-Weinberg $P=0.1366$ ), whereas the fT and $\mathrm{fC}$ was 0.708 and 0.292 for $I L 10,-819 \mathrm{~T}>\mathrm{C}$ genotype (Hardy-Weinberg $P=0.1519$ ). The fA and fG was 0.914 and 0.086 for $B C L 2,+21$ A $>\mathrm{G}$ genotype (HardyWeinberg $P=0.9549$ ), whereas the $\mathrm{fC}$ and $\mathrm{fA}$ was 0.618 and 0.322 for BCL2, $-938 \mathrm{C}>\mathrm{A}$ genotype (Hardy-Weinberg $P=0.0527)$, respectively.

The LD analysis showed a strong LD between $-819 \mathrm{~T}>\mathrm{C}$ and $-592 \mathrm{~A}>\mathrm{C}$ in IL10 SNP $\left(D^{\prime}=1.00\right)$, thus generating two haplotypes for IL10 gene polymorphism as TA (frequency $0.708)$ and CC (0.289). A weak LD was noted between +21 $\mathrm{A}>\mathrm{G}$ and $-938 \mathrm{C}>\mathrm{A}$ in BCL2 SNP $\left(D^{\prime}=0.40\right)$. The frequency in IL10 haplotypes was not different between the R-CHOP and the CHOP groups $(P=0.357)$.

Disease characteristics (including IPI, stage, performance status, age, lactate dehydrogenase elevation, or extranodal involvement) were not significantly different by the IL10 or BCL2 genotype in most cases. However, the CHOP group, -938 $\mathrm{C}$ allele (AC or CC genotype) was associated with older age $(P=0.01)$, whereas -938 AA genotype was associated with more frequent extranodal involvement. In the R-CHOP group, -938 AA genotype was associated with lower stage at presentation $(P=0.01)$.

Single-marker analysis for treatment outcomes according to the frontline therapy of R-CHOP or CHOP therapy or according to the BCL2 and IL10 gene polymorphisms. We compared treatment outcomes to determine whether BCL2 and IL10 gene polymorphisms were associated with different treatment outcomes when adding rituximab to CHOP chemotherapy. We compared the outcomes according to different genotype groups in the two stratified treatment groups (R-CHOP or CHOP group) and between the R-CHOP and CHOP therapy in the two 
Table 1. Patient characteristics and treatment protocol according to the frontline regimen

\begin{tabular}{|c|c|c|c|c|}
\hline & Overall $(n=235)$ & CHOP $(n=110)$ & R-CHOP $(n=125)$ & $\boldsymbol{P}$ \\
\hline Gender (F/M) & $104 / 131$ & $49 / 61$ & $55 / 70$ & NS \\
\hline Age $(y)$, (range) & $60(16-84)$ & $59.5(16-83)$ & $60(18-84)$ & NS \\
\hline \multicolumn{5}{|l|}{ Disease, $n(\%)$} \\
\hline Age, $\geq 60$ y $(n=232)$ & $119(51)$ & $55(50)$ & $64(53)$ & NS \\
\hline Stage $3 / 4(n=231)$ & $96(42)$ & $47(43)$ & $49(41)$ & NS \\
\hline Elevated lactate dehydrogenase & $124(53)$ & $60(55)$ & $64(52)$ & NS \\
\hline Extranodal, $>1$ site & $103(44)$ & $49(45)$ & $54(43)$ & NS \\
\hline ECOG $\geq 2(n=230)$ & $41(18)$ & $20(19)$ & $21(17)$ & NS \\
\hline \multicolumn{5}{|l|}{ IPI $(n=224), n(\%)$} \\
\hline Score $0-2$ & $147(66)$ & $65(63)$ & $82(68)$ & NS \\
\hline Score 3-5 & $77(34)$ & $38(37)$ & $39(32)$ & \\
\hline \multicolumn{5}{|l|}{ Treatment } \\
\hline Chemotherapy, n (\%) & $158(67)$ & $72(65)$ & $86(69)$ & NS \\
\hline Chemotherapy + IFRT, $n(\%)$ & $77(33)$ & $38(35)$ & $39(31)$ & NS \\
\hline Cycles of chemotherapy (range) & $6(1-10)$ & $6(1-10)$ & $6(4-8)$ & NS \\
\hline HDCT/ASCT, $n(\%)$ & $12(5)$ & $7(6)$ & $4(4)$ & NS \\
\hline \multicolumn{5}{|l|}{ Response } \\
\hline Evaluable & 227 & 106 & 121 & \\
\hline $\mathrm{CR}, n(\%)$ & $166(73)$ & $73(69)$ & $93(77)$ & NS \\
\hline $\mathrm{PR}, n(\%)$ & $32(14)$ & $17(16)$ & $15(12)$ & \\
\hline $\mathrm{SD}$ or $\mathrm{PD}, n(\%)$ & $29(13)$ & $16(15)$ & $13(11)$ & \\
\hline ORR (CR + PR), $n(\%)$ & $198(87)$ & $90(85)$ & $108(89)$ & NS \\
\hline \multicolumn{5}{|l|}{ Survival } \\
\hline Median follow-up (mo) & $22(0.5-176)$ & $22(0.5-176)$ & $21.5(1-44)$ & NS \\
\hline $2-y$ OS (\%) & $72.6 \pm 3.4$ & $70.9 \pm 4.9$ & $74.0 \pm 4.8$ & NS \\
\hline 2-y FFS (\%) & $57.2 \pm 3.8$ & $54.5 \pm 5.3$ & $59.3 \pm 5.4$ & 0.03 \\
\hline 2-y progression (\%) & $38.4 \pm 3.8$ & $41.9 \pm 5.3$ & $35.8 \pm 5.5$ & 0.01 \\
\hline
\end{tabular}

Abbreviations: NS, not significant; IFRT, involved field radiotherapy; HDCT/ASCT, high-dose chemotherapy with autologous stem cell transplantation; PR, partial response; SD, stable disease; PD, progressive disease.

stratified genotype groups $\left(B C L 2^{\text {High }} / I L 10^{\text {High }}\right.$ versus BCL2 ${ }^{\text {Low }}$ and/or IL $10^{\text {Low }}$ ).

Table 3 shows that the IL10 gene polymorphism significantly influenced the ORR in the CHOP group but not in the R-CHOP group. The ORR in the CHOP group was significantly higher in the group with $-592 \mathrm{AA}$ or AC genotype compared with those with CC genotype ( $87 \%$ versus $62 \% ; P=0.04$ ) or in the group with -819 TT or TC genotype versus those with CC genotype
( $87 \%$ versus $62 \% ; P=0.04$ ). However, no difference in ORR was observed according to IL10 gene polymorphism in the R-CHOP group. The $+21 \mathrm{~A}>\mathrm{G}$ or $-938 \mathrm{C}>\mathrm{A} B C L 2$ genotype was not associated with ORR either in the R-CHOP or CHOP group, respectively.

We also compared the CR or the ORR between the R-CHOP and the CHOP groups in each genotype group as shown in Table 3 ( $P$ values are presented as $P_{\mathrm{CR}}$ and $\left.P_{\mathrm{ORR}}\right)$. A

Table 2. Frequencies of the $B C L 2$ and $I L 10$ genotypes

\begin{tabular}{|c|c|c|c|c|c|}
\hline \multirow[t]{2}{*}{ IL10 } & & \multicolumn{4}{|c|}{$-819 T>C$} \\
\hline & & CC & CT & TT & Total $n(\%)$ \\
\hline \multirow[t]{4}{*}{$-592 A>C *$} & $\mathrm{CC}$ & 23 & 0 & 0 & $23(11)$ \\
\hline & CA & 0 & 78 & 0 & $78(36)$ \\
\hline & AA & 0 & 1 & 112 & $113(53)$ \\
\hline & Total $n(\%)$ & $23(11)$ & 79 (37) & $112(52)$ & \\
\hline \multirow[t]{2}{*}{$B C L 2$} & & \multicolumn{4}{|c|}{$-938 \mathrm{C}>\mathrm{A}^{\dagger}$} \\
\hline & & AA & AC & CC & Total n (\%) \\
\hline \multirow[t]{4}{*}{$+21 A>G$} & AA & 20 & 63 & 96 & $179(84)$ \\
\hline & AG & 8 & 18 & 7 & 33 (15) \\
\hline & GG & 0 & 0 & 2 & $2(1)$ \\
\hline & Total $n(\%)$ & $28(13)$ & $81(40)$ & 105 (49) & \\
\hline
\end{tabular}

$*_{n}=126$, CC (23), CA (79), AA (114), 2 cases missed due to lack of genotype for IL10, -819 T>C.

${ }^{\dagger} n=126, \mathrm{AA}(29), \mathrm{AC}(81), \mathrm{CC}(106), 2$ cases missed due to lack of genotype for $B C L 2,+21 \mathrm{~A}>\mathrm{G}$. 
Table 3. Response to CHOP or R-CHOP chemotherapy according to the IL1O or BCL2 genotypes

\begin{tabular}{|c|c|c|c|c|c|c|c|c|c|}
\hline & & \multicolumn{4}{|c|}{ IL10 } & \multicolumn{4}{|c|}{ BCL2 } \\
\hline & & \multicolumn{2}{|c|}{$-592 A>C$} & \multicolumn{2}{|c|}{$-819 T>C$} & \multicolumn{2}{|c|}{$+21 A>G$} & \multicolumn{2}{|c|}{$-938 C>A$} \\
\hline & & AA/AC & CC & TT/TC & $\mathbf{C C}$ & GG/GA & AA & CC/CA & AA \\
\hline & & $n=82$ & $n=13$ & $n=82$ & $n=13$ & $n=17$ & $n=81$ & $n=85$ & $n=15$ \\
\hline \multirow[t]{5}{*}{$\mathrm{CHOP}$} & CR & $56(68)$ & $8(62)$ & $56(68)$ & $8(62)$ & $12(71)$ & $54(67)$ & $56(66)$ & $12(80)$ \\
\hline & PR & $15(18)$ & $0(0)$ & $15(18)$ & $0(0)$ & $4(24)$ & $13(16)$ & $15(18)$ & $2(13)$ \\
\hline & $S D+P D$ & 11 (13) & $5(38)$ & $11(13)$ & $5(38)$ & $1(6)$ & 14 (17) & $14(16)$ & $1(7)$ \\
\hline & $P$ & \multicolumn{2}{|c|}{0.04} & \multicolumn{2}{|c|}{0.04} & \multicolumn{2}{|c|}{0.4} & \multicolumn{2}{|c|}{0.5} \\
\hline & & $n=105$ & $n=9$ & $n=103$ & $n=9$ & $n=17$ & $n=91$ & $n=94$ & $n=14$ \\
\hline \multirow[t]{4}{*}{$\mathrm{R}-\mathrm{CHOP}$} & CR & $82(78)$ & $8(89)$ & $81(78)$ & $8(89)$ & $12(71)$ & $70(77)$ & $72(77)$ & $10(71)$ \\
\hline & PR & $13(13)$ & $1(11)$ & $12(12)$ & $1(11)$ & $3(18)$ & $11(12)$ & 12 (13) & $2(14)$ \\
\hline & $S D+P D$ & $10(9)$ & $0(0)$ & $10(10)$ & $0(0)$ & $2(12)$ & $10(11)$ & $10(11)$ & $2(14)$ \\
\hline & $P$ & \multicolumn{2}{|c|}{0.6} & \multicolumn{2}{|c|}{0.6} & \multicolumn{2}{|c|}{0.8} & \multicolumn{2}{|c|}{0.9} \\
\hline$P_{\mathrm{CR}} *$ & & 0.1 & 0.2 & 0.1 & 0.2 & 1.0 & 0.1 & 0.1 & 0.6 \\
\hline$P_{\mathrm{ORR}^{\dagger}}{ }^{+}$ & & 0.4 & 0.05 & 0.4 & 0.05 & 1.0 & 0.3 & 0.3 & 0.5 \\
\hline
\end{tabular}

${ }^{*} P_{\mathrm{CR}}, P$ value for difference of $\mathrm{CR}$ rate between $\mathrm{R}-\mathrm{CHOP}$ and $\mathrm{CHOP}$ groups confined to each genotype group.

${ }^{\dagger} P_{\mathrm{ORR}}, P$ value for difference of ORR between R-CHOP and CHOP groups confined to each genotype group.

significantly improved ORR in the R-CHOP group was noted compared with the CHOP group in a subgroup with the -592 CC genotype $(P=0.05)$ or with the -819 CC genotype $(P=0.05)$.

Better FFS and OS in each IL10 genotype group was not detected in overall patients or in the R-CHOP group. Different OS trends were noted in the CHOP group according to the IL10, $-592 \mathrm{~A}>\mathrm{C}(P=0.06)$ or $-819 \mathrm{~T}>\mathrm{C}$ genotypes $(P=0.06)$. The R-CHOP group had better FFS in the groups with $-592 \mathrm{AA} /$ AC $(P=0.03),-819$ TT/TC $(P=0.02)$, and IL10 genotype, +21 AA $(P=0.05)$. In addition, better FFS was noted in the group with 938 AA BCL2 genotypes among R-CHOP-received patients $(P=0.05)$ probably due to more frequent presentation with lower stage disease.

IL10 gene haplotype associates with treatment outcomes following R-CHOP or CHOP therapy. The analysis revealed a strong association of the IL10 haplotype with ORR in the CHOP group but not in the R-CHOP group. The group with 0 or 1 copy of the CC haplotype (TA/TA or TA/CC haplotype) showed a $90 \%$ of ORR (71 of 79 patients) compared with $67 \%$ of ORR in those with 2 copies of the CC haplotype (10 of 15 patients; $P=0.03$ ) when confined to the CHOP group. This confirmed the negative prognostic effect of the CC IL10 haplotype on survival in DLBCL patients. However, this negative effect was not noted in the R-CHOP group $(P=0.3)$. It is possible that R-CHOP therapy could overcome negative effects of the CC IL10 haplotype on prognosis in DLBCL patients.

Multivariate analyses for response to CHOP or R-CHOP therapy. Further analyses with multiple logistic regression was conducted to identify the predictive factors of ORR and identified the CC IL10 haplotype as the only independent predictive factor for ORR in the overall population (odds ratio, 3.12; 95\% CI, 1.01-9.65; $P=0.05)$. We performed these analyses separately in either R-CHOP or CHOP group and the influence of the IL10 gene haplotype was more prominent in the CHOP group (OR, 6.07; 95\% CI, 1.56-23.69; $P=0.009$ ) but not significant in the R-CHOP group.
Interaction between IL10 and BCL2 gene polymorphisms and its effect on the treatment outcomes of DLBCL patients. We generated two risk groups according to patient SNP information on the IL10 haplotype and BCL2 genotype to evaluate interactions between the IL10 and BCL2 gene polymorphisms: (a) high BCL2 and high IL10 inducible group with both -938 AA BCL2 genotypes and 2 copies of the CC IL10 haplotype for loci $-819 \mathrm{~T}>\mathrm{C}$ and $-592 \mathrm{~A}>\mathrm{C}\left(B C L 2^{\mathrm{High}} / \mathrm{IL} 10^{\mathrm{High}}\right)$ and $(b)$ low BCL2 or low IL10 inducible group with -938 AC/CC BCL2 genotype or 0 to 1 copy of the CC IL10 haplotype for loci -819 $\mathrm{T}>\mathrm{C}$ and $-592 \mathrm{~A}>\mathrm{C}\left(B C L 2^{\text {Low }}\right.$ and/or $\left.I L 10^{\mathrm{Low}}\right)$.

Frontline therapy-stratified subgroup analysis was done in each group who received either CHOP or R-CHOP therapy according to their genotypes. No significant differences were noted in OS as shown in Fig. 1A. The group with $B C L 2^{\text {Low }}$ and/or $I L 10^{\text {Low }}$ showed better FFS than those with $B C L 2^{\mathrm{High}} / I L 10^{\mathrm{High}}$ in the CHOP group $(P=0.002)$ but not in the R-CHOP group $(P=0.354$; Fig. $1 \mathrm{~B})$. The survival benefits of $\mathrm{R}$-CHOP were prominent in the subgroup with $B C L 2^{\mathrm{High}} /$ IL $10^{\text {High }}$ over CHOP therapy in terms of FFS $(P=0.012$; Fig. 1B). The R-CHOP group showed a lower risk of progression compared with the CHOP group in the subgroup with $B C L 2^{\text {High }} / I L 10^{\text {High }}(P=0.004 ;$ Fig. $1 \mathrm{C})$ but not in other genotypes (BCL2 ${ }^{\text {Low }}$ and/or $I L 10^{\text {Low }} P=0.167$ ). R-CHOP therapy might overcome a high IL-10 and bcl-2 milieu, which are poor prognostic factors for DLBCL and neutralize the unfavorable effects of high IL-10 and bcl-2 environment. Figure 2 summarizes the $\mathrm{HR}$ and 95\% CIs according to the frontline therapy and the BCL2/IL10 genotype in overall population and in either treatment-stratified or genotypestratified subgroups.

The treatment-stratified subgroup analysis suggested a trend of better outcomes in the R-CHOP therapy group compared with the CHOP therapy group in the overall population with respect to OS (HR, 0.59; 95\% CI, 0.33-1.08), FFS (HR, 0.60; $95 \% \mathrm{CI}, 0.37-0.97$ ), and risk of progression (HR, 0.55; 95\% CI, 0.33-0.91; Fig. 2A). The benefit had greater significance, especially in the group with the BCL2 ${ }^{\mathrm{High}} / \mathrm{IL} 10^{\mathrm{High}}$ genotype 


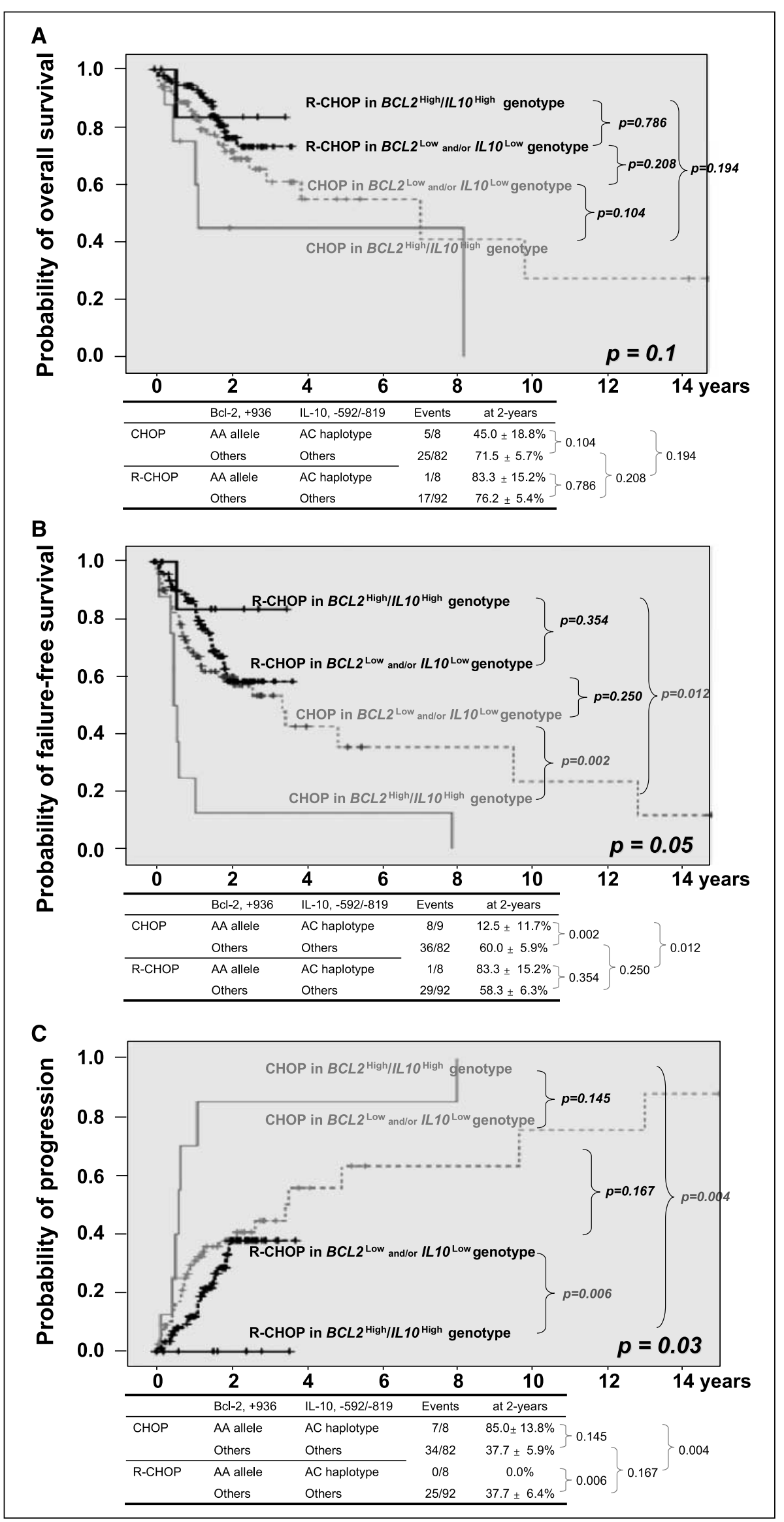

Fig. 1. Survival analysis of the patients divided into four groups according to their frontline regimen ( $\mathrm{R}-\mathrm{CHOP}$ versus $\mathrm{CHOP}$ regimen) plus the interaction of IL10/BCL2 gene polymorphisms: $(A) \mathrm{OS},(B) \mathrm{FFS}$, and $(C)$ probability of progression 
Fig. 2. Comparison of HRs according to the frontline therapy (R-CHOP versus CHOP therapy) after stratification of the patients by the BCL2/IL10 genotype, according to the $B C L 2 / I L 10$ genotype after stratification of the patients by frontline therapy ( $\mathrm{R}-\mathrm{CHOP}$ versus $\mathrm{CHOP}$ ), and according to the $B C L 2 / / L 10$ genotype. $A$, favorable outcomes from R-CHOP compared with $\mathrm{CHOP}$ was observed in the overall population or in the subgroup with the $B C L 2^{\text {High }} / I L 10^{\text {High }}$ genotype in terms of FFS but not in the patients with the $B C L 2^{\text {Low }}$ and/or $/ L 10^{\text {Low }}$ genotypes. $B$, unfavorable outcomes from the $B C L 2^{\text {High }} /$ IL10 $10^{\text {High }}$ genotype were prominent, particularly in the CHOP group. However, this effect was neutralized following the R-CHOP therapy.

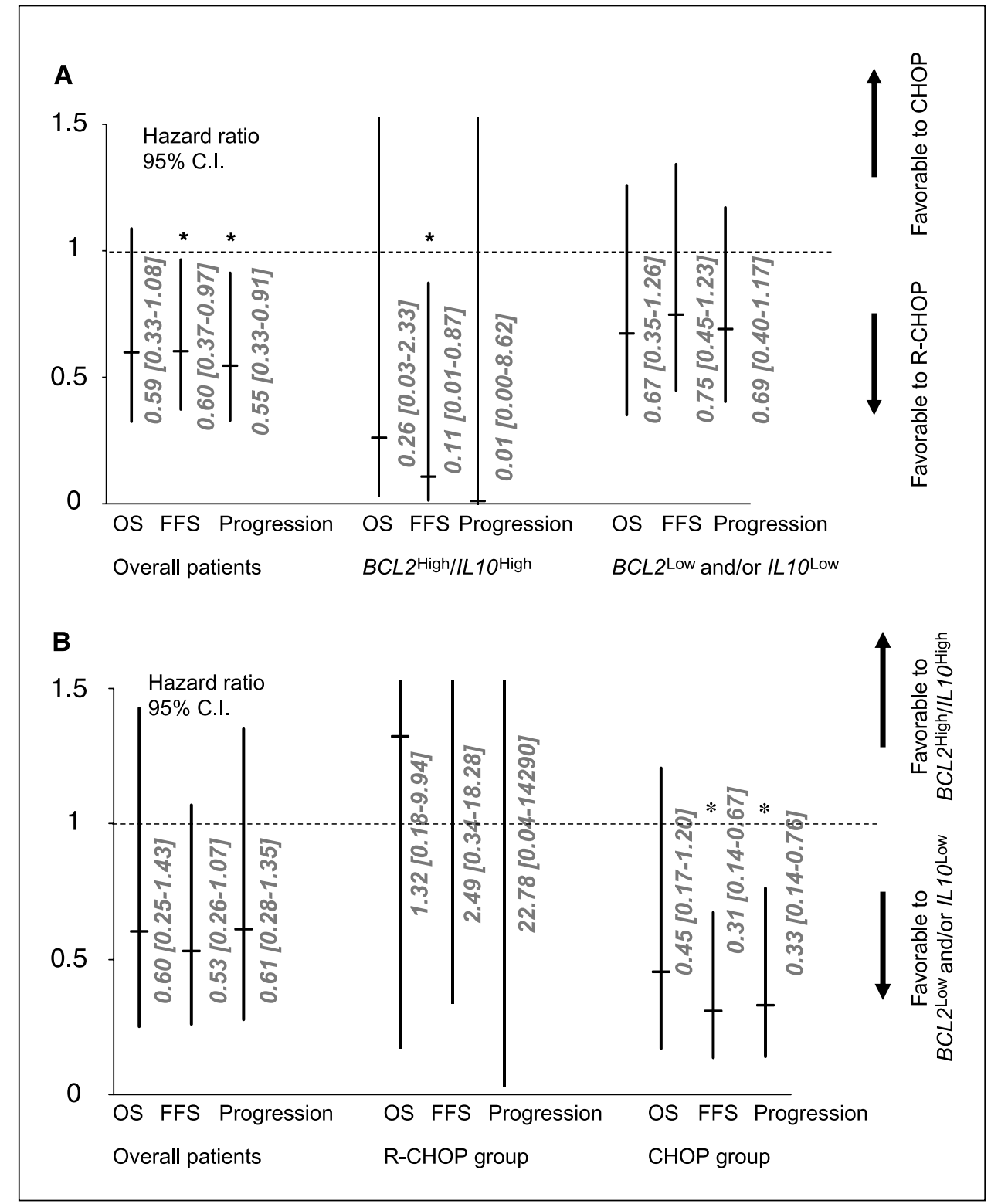

with respect to FFS. The HR of patients for FFS in patients who received $\mathrm{R}-\mathrm{CHOP}$ was 0.11 (95\% CI, 0.01-0.87).

Meanwhile, this effect was not significant in the group with $B C L 2^{\text {Low }}$ and/or $I L 10^{\text {Low }}$ genotypes as seen by the HR of RCHOP for OS (HR, 1.32; 95\% CI, 0.18-9.94), FFS (HR, 2.49; 95\% CI, 0.34-13.28), and risk of progression (HR, 22.78; 95\% CI, 0.04-14.29; Fig. 2B). There was a possible trend toward unfavorable effects of the $B C L 2^{\mathrm{High}} / \mathrm{IL} 10^{\mathrm{High}}$ genotype on survival in the overall population, but this was not statistically significant (Fig. 2B). In addition, the unfavorable effects of the $B C L 2^{\text {High }} / I L 10^{\text {High }}$ genotype were prominent in the CHOP group with respect to FFS (HR, 0.31; 95\% CI, 0.14-0.67) and with respect to the risk of progression (HR, 0.33; 95\% CI, $0.14-0.76)$. However, the unfavorable effect of the BCL2 ${ }^{\mathrm{High}} /$ IL10 ${ }^{\text {High }}$ genotype has shown to be neutralized in the R-CHOP group (Fig. 2B).

Multivariate survival analyses considering the interaction between IL10 and BCL2 gene polymorphism. Multivariate analysis in the overall population confirmed that the IPI and frontline treatment regimens are independent prognostic factors for OS, FFS, and progression. However, the effect of the IL10/BCL2 gene polymorphism was not evident (Table 4). Multivariate analysis was done in the frontline treatmentstratified subgroup analysis (each subgroup received either CHOP or R-CHOP therapy). The group with BCL2 ${ }^{\mathrm{High}} / \mathrm{IL} 10^{\mathrm{High}}$ in the CHOP group had an independent risk factor for FFS (HR, 2.94; 95\% CI, 1.27-6.76; $\left.P=0.01 ; P_{\text {corr }}=0.04\right)$ together with IPI $(P<0.001)$ and for the probability of progression (HR, 3.21; 95\% CI, 1.46-7.04; $\left.P=0.004 ; P_{\text {corr }}=0.016\right)$ together with IPI $(P<0.001)$. Poor prognostic implications in the $B C L 2^{\mathrm{High}} / \mathrm{IL} 10^{\mathrm{High}}$ genotype in the R-CHOP group were neutralized for FFS or progression.

Taken together, these results suggest that rituximab may be helpful to overcome the IL-10/bcl-2-mediated resistance in DLBCL especially in the group with $B C L 2^{\mathrm{High}} / \mathrm{IL} 10^{\mathrm{High}}$ genotype. In addition, the current data suggest the interaction 
between IL10 and BCL2 gene polymorphisms, which works as unfavorable risk factor in DLBCL patients when treated with CHOP chemotherapy without adding rituximab.

\section{Discussion}

The current study suggests that the benefits derived through the addition of rituximab to CHOP chemotherapy was potentiated in the patient subgroup with the BCL2 ${ }^{\mathrm{High}} / \mathrm{IL} 10^{\mathrm{High}}$ genotype compared with patients with the $B C L 2^{\text {Low }}$ and/or IL $10^{\text {Low }}$ genotypes, particularly in terms of FFS or the probability of progression. These data suggested a potential interaction between bcl-2 and IL-10 as a target of R-CHOP therapy in overcoming chemoresistance in patients with DLBCL.

Higher expression of bcl-2 and/or IL-10 in some patients with DLBCL can partially explain the mechanism of treatment failure of CHOP therapy. Conversely, blocking this pathway with rituximab can be a potential mechanism of its therapeutic action. The current study highlights the effect of interaction of BCL2/IL10 polymorphisms on different treatment outcomes between patients receiving R-CHOP and patients receiving CHOP therapy. The patients with the $B C L 2^{\mathrm{High}} / \mathrm{IL} 10^{\mathrm{High}}$ genotype, whose prognosis was predicted to be worse than in individuals with $B C L 2^{\text {Low }}$ and/or $I L 10^{\text {Low }}$ genotypes, showed significantly worse FFS and higher risks of progression in the subgroup treated with CHOP therapy. This was not seen in patients treated with R-CHOP therapy. This finding suggested that R-CHOP therapy could neutralize the negative prognostic effect of the BCL2 $2^{\mathrm{High}} / \mathrm{IL} 10^{\mathrm{High}}$ genotype on DLBCL survival.

The BCL2 gene, located on chromosome 18q21.3, consists of 3 exons and 2 promoters. We examined the -938 C >A BCL2 genotype, which was shown to be correlated with bcl-2 expression and to be associated with disease progression and survival in chronic lymphocytic leukemia (17). The -938 C>A polymorphism is located in P2 promoter region, which functions as a negative regulatory element to the P1 promoter in BCL2 gene expression $(28,29)$. Accordingly, the -938 CC allele can increase the production of negative regulatory elements to the P1 promoter, resulting in down-regulation of the bcl-2 expression (17). In addition, we included the +21 $\mathrm{A}>\mathrm{G}$ BCL2 and $-938 \mathrm{C}>\mathrm{A}$ BCL2 genotypes together based on previous results, suggesting a LD between the $-938 \mathrm{C}>\mathrm{A}$ and the +21 A $>$ G genotypes (30). The linkage coefficient between -938 and +21 was too weak $\left(D^{\prime}=0.40\right)$ to generate a haplotype in our study.

The IL10 promoter gene polymorphism has been identified as an independent prognostic factor for progression-free survival or OS in DLBCL patients receiving CHOP-based chemotherapy (9). Several haplotype studies for the IL10 promoter gene suggested that the ATA haplotype for -1082, -819 , and -592 loci was associated with low IL-10 inducibility $(23-26)$. In the present study, the group with high IL-10 inducibility had a higher ORR or better FFS than the other group when treated with CHOP therapy but not with R-CHOP therapy. However, this effect was not confirmed in the multivariate analysis based on Cox's proportional hazards model.

Recent studies have emphasized the significance of SNP-toSNP interaction during interpretation of SNP data. The effect of SNPs cannot be detected in single marker analyses but became apparent when interpretation considered SNP-to-SNP interaction. Accordingly, the interaction among candidate genes needs to be considered in the analysis of the data $(31,32)$. For example, MDM2 SNP309 and TP53 $\mathrm{Arg}^{72}$ Pro interact to alter the susceptibility of therapy-related acute myeloid leukemia (33), and neither polymorphism alone influenced the risk of therapy-related acute myeloid leukemia. Similarly, we have taken identified the interaction between the IL10 gene haplotype and the $-938 \mathrm{C}>\mathrm{A}$ BCL2 genotype, and have generated the $B C L 2^{\mathrm{High}} / \mathrm{IL} 10^{\mathrm{High}}$ genotype group and the $B C L 2^{\text {Low }}$ and/or $I L 10^{\text {Low }}$ genotype group.

Mounier et al. (8) revealed that a mechanism of action in R-CHOP therapy is negation of bcl-2-mediated chemoresistance in DLBCL. The group with high bcl-2 expression showed a worse treatment outcome compared with those with low bcl-2 expression in individuals with $\mathrm{CHOP}$ therapy. The negative effect of bcl-2 expression was neutralized with R-CHOP therapy, showing similar treatment outcomes between RCHOP-treated and CHOP-treated groups.

Table 4. Multivariate analyses of the prognostic factors for OS/FFS and probability of progression

\begin{tabular}{|c|c|c|c|c|}
\hline Group & Variables & Variables & $\boldsymbol{P}$ & HR $(95 \% \mathrm{CI})$ \\
\hline \multirow[t]{5}{*}{ Overall* } & Progression & Intermediate-to-high or high IPI (vs low/low-to-intermediate) & $<0.001$ & $3.32(1.99-5.54)$ \\
\hline & & CHOP therapy (vs R-CHOP) & 0.05 & $2.99(1.64-5.49)$ \\
\hline & FFS & Intermediate-to-high or high IPI (vs low/low-to-intermediate) & $<0.001$ & $3.19(1.98-5.17)$ \\
\hline & & CHOP therapy (vs R-CHOP) & 0.03 & $1.79(1.07-2.98)$ \\
\hline & os & Intermediate-to-high or high IPI (vs low/low-to-intermediate) & $<0.001$ & $3.84(2.07-7.12)$ \\
\hline \multirow[t]{3}{*}{$\mathrm{R}-\mathrm{CHOP}{ }^{\dagger}$} & Progression & Intermediate-to-high or high IPI (vs low/low-to-intermediate) & 0.001 & $3.61(1.72-7.52)$ \\
\hline & FFS & Intermediate-to-high or high IPI (vs low/low-to-intermediate) & $<0.001$ & $4.39(1.92-10.00)$ \\
\hline & os & Intermediate-to-high or high IPI (vs low/low-to-intermediate) & 0.003 & $4.12(1.60-10.64)$ \\
\hline \multirow[t]{5}{*}{$\mathrm{CHOP}^{\dagger}$} & Progression & Intermediate-to-high or high IPI (vs low/low-to-intermediate) & $<0.001$ & $3.21(1.70-6.06)$ \\
\hline & & $-938 A A, B C L 2+0 / 1$ copy of CC ht, IL10 (vs others) & $0.004^{\ddagger}$ & $3.21(1.46-7.04)$ \\
\hline & FFS & Intermediate-to-high or high IPI (vs low/low-to-intermediate) & $<0.001$ & $3.52(1.81-6.85)$ \\
\hline & & $-938 A A, B C L 2+0 / 1$ copy of $C C$ ht, ILIO (vs others) & $0.01^{\ddagger}$ & $2.94(1.27-6.76)$ \\
\hline & OS & Intermediate-to-high or high IPI (vs low/low-to-intermediate) & 0.001 & $3.70(1.68-8.20)$ \\
\hline
\end{tabular}

*IPI, frontline chemotherapy, and BCL2/IL10 polymorphism were included in the final model.

${ }^{\dagger}$ IPI and $B C L 2 / I L 10$ polymorphism were included in the final model.

$\ddagger P_{\text {corr }}$ (Bonferroni-corrected $P$ value $)=0.016$ for progression and 0.04 for FFS in CHOP group. 
Marked benefits from the R-CHOP patients in terms of FFS were observed in the group with the BCL $2^{\mathrm{High}} / I L 10^{\mathrm{High}}$ genotype. Both bcl-2 and IL-10 expression, particularly with CHOP chemotherapy, were well-known unfavorable prognostic factors in DLBCL patients. Therefore, we expected this group to have the worst clinical prognosis, and their treatment outcome in the overall population or in the CHOP group was worse than those with BCL $2^{\text {Low }}$ and/or $I L 10^{\text {Low }}$ genotype. However, we showed neutralizing effects of R-CHOP on the poor outcome of patients with the BCL2 ${ }^{\mathrm{High}} / \mathrm{IL} 10^{\mathrm{High}}$ genotype.

In addition, the interaction between BCL2 and IL10 polymorphisms on improvement of outcomes in R-CHOP therapy suggests that that rituximab could overcome drug resistance in DLBCL by blocking the IL-10/bcl-2-mediated loops. The IL-10/ bcl-2-mediated pathway may not explain the entire mechanism of action of R-CHOP toward DLBCL. Cerhan et al. (34) showed that the multiple candidate gene polymorphism approach could stratify patients with follicular lymphoma according to their prognosis; therefore, further study will help direct the multiple candidate gene polymorphism approach in targeting multiple potential drug resistance pathways in DLBCL.

The interpretation of the current data requires special caution. The current study has been done with a small number of patients and with a short duration of follow-up. Accordingly, validation of the current results will be needed in patients with different ethnicities to contribute to clear conclusions on the issue.

In conclusion, our data suggested that an interactive effect between BCL2 and IL10 SNPs was significant in the group with both -938 AA BCL2 genotype and 1 to 2 copies of CC IL10 haplotype, especially in terms of FFS and progression, and that chemotherapy resistance in DLBCL may involve interactions between BCL2 and IL10 genes, which could be overcome by the use of R-CHOP.

\section{Disclosure of Potential Conflicts of Interest}

No potential conflicts of interest were disclosed.

\section{References}

1. Coiffier B, Lepage E, Briere J, et al. CHOP chemotherapy plus rituximab compared with $\mathrm{CHOP}$ alone in elderly patients with diffuse large-B-cell lymphoma. N Engl J Med 2002;346:235-42.

2. Coiffier B. Rituximab in combination with $\mathrm{CHOP}$ improves survival in elderly patients with aggressive non-Hodgkin's Iymphoma. Semin Oncol 2002;29: $18-22$.

3. Sehn LH, Donaldson J, Chhanabhai M, et al. Introduction of combined CHOP plus rituximab therapy dramatically improved outcomes of diffuse large Bcell lymphoma in British Columbia. J Clin Oncol 2005;23:5027-33.

4. Ozdemir F, Aydin F, Yilmaz M, et al. The effects of IL-2, IL-6 and IL-10 levels on prognosis in patients with aggressive non-Hodgkin's lymphoma (NHL). J Exp Clin Cancer Res 2004;23:485-8.

5. Stasi R, Zinzani PL, Galieni $P$, et al. Prognostic value of serum IL-10 and soluble IL-2 receptor levels in aggressive non-Hodgkin's lymphoma. Br J Haematol 1994;88:770-7.

6. Cartron G, Watier H, Golay J, Solal-Celigny P. From the bench to the bedside: ways to improve rituximab efficacy. Blood 2004;104:2635-42.

7. Mounier N, Briere J, Gisselbrecht C, Reyes F, Gaulard $\mathrm{P}$, Coiffier B. Estimating the impact of rituximab on bcl-2-associated resistance to $\mathrm{CHOP}$ in elderly patients with diffuse large B-cell lymphoma. Haematologica 2006;91:715-6.

8. Mounier N, Briere J, Gisselbrecht C, et al. Rituximab plus $\mathrm{CHOP}$ (R-CHOP) overcomes bcl-2-associated resistance to chemotherapy in elderly patients with diffuse large B-cell lymphoma (DLBCL). Blood 2003; 101:4279-84.

9. Lech-Maranda E, Baseggio L, Bienvenu J, et al. Interleukin-10 gene promoter polymorphisms influence the clinical outcome of diffuse large B-cell lymphoma. Blood 2004;103:3529-34

10. el-Far M, Fouda M, Yahya R, el-Baz H. Serum IL-10 and IL-6 levels at diagnosis as independent predictors of outcome in non-Hodgkin's lymphoma. J Physiol Biochem 2004;60:253-8.

11. Aydin F, Yilmaz M, Ozdemir F, Kavgaci H, Yavuz MN, Yavuz AA. Correlation of serum IL-2, IL-6 and IL-10 levels with international prognostic index in patients with aggressive non-Hodgkin's lymphoma. Am J Clin Oncol 2002;25:570-2.
12. Weber-Nordt RM, Henschler R, Schott E, et al. Interleukin-10 increases bcl-2 expression and survival in primary human $\mathrm{CD} 34^{+}$hematopoietic progenitor cells. Blood 1996;88:2549-58.

13. Voorzanger N, Touitou $R$, Garcia $E$, et al. Interleukin (IL) -10 and IL- 6 are produced in vivo by non-Hodgkin's lymphoma cells and act as cooperative growth factors. Cancer Res 1996:56:5499-505.

14. Alas S, Emmanouilides $C$, Bonavida B. Inhibition of interleukin 10 by rituximab results in down-regulation of bcl-2 and sensitization of B-cell non-Hodgkin's lymphoma to apoptosis. Clin Cancer Res 2001;7: 709-23.

15. Alas S, Bonavida B. Rituximab inactivates signal transducer and activation of transcription 3 (STAT3) activity in B-non-Hodgkin's lymphoma through inhibition of the interleukin 10 autocrine/paracrine loop and results in down-regulation of $\mathrm{Bcl}-2$ and sensitization to cytotoxic drugs. Cancer Res 2001;61:5137-44.

16. Alas S, Bonavida B. Inhibition of constitutive STAT3 activity sensitizes resistant non-Hodgkin's lymphoma and multiple myeloma to chemotherapeutic drugmediated apoptosis. Clin Cancer Res 2003:9:316 - 26. 17. Nuckel H, Frey UH, Bau M, et al. Association of a novel regulatory polymorphism $(-938 \mathrm{C}) \mathrm{A})$ in the BCL2 gene promoter with disease progression and survival in chronic lymphocytic leukemia. Blood 2007;109:290-7.

18. Park YH, Lee JJ, Ryu MH, et al. Improved therapeutic outcomes of DLBCL after introduction of rituximab in Korean patients. Ann Hematol 2006;85:257-62.

19. Kim DH, Lee NY, Sohn SK, et al. IL-10 promoter gene polymorphism associated with the occurrence of chronic GVHD and its clinical course during systemic immunosuppressive treatment for chronic GVHD after allogeneic peripheral blood stem cell transplantation. Transplantation 2005;79:1615-22.

20. Cheson BD, Horning SJ, Coiffier B, et al. Report of an international workshop to standardize response criteria for non-Hodgkin's lymphomas. NCISponsored InternationalWorking Group. J Clin Oncol 1999;17:1244.

21. Barrett JC, Fry B, Maller J, Daly MJ. Haploview: analysis and visualization of LD and haplotype maps. Bioinformatics 2005;21:263-5.

22. Stephens M, Smith N, Donnelly P. A new statistical method for haplotype reconstruction from population data. Am J Hum Gen 2001;68:978-89.
23. Crawley E, Kay R, Sillibourne J, Patel P, Hutchinson I. Woo P. Polymorphic haplotypes of the interleukin-10 $5^{\prime}$ flanking region determine variable interleukin-10 transcription and are associated with particular phenotypes of juvenile rheumatoid arthritis. Arthritis Rheum 1999;42:1101 - 8

24. Hulkkonen J, Pertovaara M, Antonen J, Lahdenpohja N, Pasternack A, Hurme M. Genetic association between interleukin-10 promoter region polymorphisms and primary Sjogren's syndrome. Arthritis Rheum 2001;44:176-9.

25. Karjalainen J, Hulkkonen J, Nieminen MM, et al. Interleukin-10 gene promoter region polymorphism is associated with eosinophil count and circulating immunoglobulin E in adult asthma. Clin Exp Allergy 2003;33:78-83

26. Lim S, Crawley E, Woo P, Barnes PJ. Haplotype as sociated with low interleukin-10 production in patients with severe asthma. Lancet 1998;352:113

27. Rady PL, Matalon R, Grady J, et al. Comprehensive analysis of genetic polymorphisms in the interleukin-10 promoter: implications for immune regulation in specific ethnic populations. Genet Test 2004;8:194-203.

28. Young RL, Korsmeyer SJ. A negative regulatory element in the bcl-2 $5^{\prime}$-untranslated region inhibits expression from an upstream promoter. Mol Cell Bio 1993;13:3686-97.

29. Seto $M$, Jaeger $U$, Hockett RD, et al. Alternative promoters and exons, somatic mutation and deregulation of the $\mathrm{Bcl}-2-\lg$ fusion gene in lymphoma. EMBO J 1988; 7:123-31.

30. Park BL, Kim LH, Cheong HS, et al. Identification of variants in cyclin D1 (CCND1) and B-cell CLL/lymphoma 2 (BCL2). J Hum Genet 2004:49:449-54.

31. Onay VU, Briollais L, Knight JA, et al. SNP-SNP interactions in breast cancer susceptibility. BMC Cance 2006;6:114.

32. Goodman JE, Mechanic LE, Luke BT, Ambs S, Chanock S, Harris CC. Exploring SNP-SNP interactions and colon cancer risk using polymorphism interaction analysis. Int J Cancer 2006;118:1790-7.

33. Ellis NA, Huo D, Yildiz O, et al. MDM2 SNP309 and TP53 $\mathrm{Arg}^{72}$ Pro interact to alter therapy-related acute myeloid leukemia susceptibility. Blood 2008.

34. Cerhan JR,Wang S, Maurer MJ, et al. Prognostic significance of host immune gene polymorphisms in follicular lymphoma survival. Blood 2007;109:5439-46. 


\section{Clinical Cancer Research}

\section{Interaction between BCL2 and Interleukin-10 Gene Polymorphisms Alter Outcomes of Diffuse Large B-Cell Lymphoma following Rituximab Plus CHOP Chemotherapy}

Yeon Hee Park, Sang Kyun Sohn, Jong Gwang Kim, et al.

Clin Cancer Res 2009;15:2107-2115.

Updated version Access the most recent version of this article at:

http://clincancerres.aacrjournals.org/content/15/6/2107

Supplementary Access the most recent supplemental material at:

Material http://clincancerres.aacrjournals.org/content/suppl/2009/03/13/1078-0432.CCR-08-1588.DC1 .html

Cited articles This article cites 33 articles, 15 of which you can access for free at:

http://clincancerres.aacrjournals.org/content/15/6/2107.full.html\#ref-list-1

Citing articles This article has been cited by 3 HighWire-hosted articles. Access the articles at:

http://clincancerres.aacrjournals.org/content/15/6/2107.full.html\#related-urls

E-mail alerts Sign up to receive free email-alerts related to this article or journal.

Reprints and To order reprints of this article or to subscribe to the journal, contact the AACR Publications Subscriptions Department at pubs@aacr.org.

Permissions To request permission to re-use all or part of this article, contact the AACR Publications Department at permissions@aacr.org. 